\title{
Cardiac resynchronisation was effective for moderate to severe heart failure with intraventricular conduction delay
}

\author{
Abraham WT, Fisher WG, Smith AL, et al, for the MIRACLE Study Group. Cardiac resynchronisation in chronic heart \\ failure. N Engl J Med. 2002;346:1845-53.

\section{QUESTION: In patients with moderate to severe heart failure and an intraventricular conduction delay, does cardiac resynchronisation improve clinical outcomes?}

\section{Design}

Randomised (unclear allocation concealment*), blinded (unclear),* controlled trial with 6 month follow up (Multicenter InSync Randomised Clinical Evaluation [MIRACLE]).

\section{Setting}

45 centres in the United States and Canada.

\section{Patients}

453 patients (mean age $64 \mathrm{y}, 68 \%$ men) who had moderate or severe (New York Heart Association [NYHA] functional class III or IV) chronic heart failure resulting from ischaemic or non-ischaemic cardiomyopathy; received stabilised doses of medication for $\geq 1$ month ( $\geq 3$ mo for $\beta$-blockers); and had left ventricular ejection fraction $\leq 35 \%$, a QRS interval $\geq 130 \mathrm{msec}$, and a 6 minute walking distance $\leq 450 \mathrm{~m}$. Exclusion criteria included use of pacemakers or cardioverterdefibrillators, a cardiac or cerebral ischaemic event in the previous 3 months, and atrial arrhythmia in the previous month. Follow up was $\geq 90 \%$ for all outcomes.

\section{Intervention}

All patients received implantation of a cardiacresynchronisation device (InSync model 8040, Medtronic, Minneapolis, MN, USA) with 3 pacing leads: a standard right atrial lead, a standard right ventricular lead, and a specialised left ventricular lead. Patients were allocated to atrial-synchronised biventricular pacing $(\mathrm{n}=$ 228) or no pacing $(\mathrm{n}=225)$.

Source of funding: Medtronic

\section{Main outcome measures}

Change in NYHA class, death or worsening heart failure, quality of life (Minnesota Living with Heart Failure score), and distance walked in 6 minutes.

\section{Main results}

Analysis was by intention to treat. More patients in the resynchronisation group than in the no-pacing group
For correspondence: Dr WT Abraham, University of Kentuck

Lexingtor

Email

wtabra2@uky.edu. had an improvement in NYHA class. Resynchronisation led to fewer patients who died or had worsening of heart failure. Patients in the resynchronisation group had a greater improvement in quality of life (median score change $-18 v-9, \mathrm{p}=0.001)$ and were able to walk further in 6 minutes (median change in distance from baseline $39 v 10 \mathrm{~m}, \mathrm{p}=0.005)$ than patients in the no-pacing group.

\section{Conclusion}

In patients with moderate to severe heart failure and an intraventricular conduction delay, cardiac resynchronisation reduced death or worsening of heart failure and improved quality of life and walking ability.

*See glossary.

\section{COMMENTARY}

The MIRACLE trial shows that 1 in 5 patients with advanced symptomatic congestive heart failure and an intraventricular conduction delay have incremental improvement of $\geq 1$ NYHA symptom class at 6 months with biventricular pacing. Limited information is available at present to predict the subset of patients with congestive heart failure and an intraventricular conduction delay who will benefit from resynchronisation pacing therapy. Given the relatively low rate of short term response to biventricular pacing, the more mundane initial management efforts at correcting modifiable underlying factors (eg, ischaemia and valvular disease) and optimising medical treatment should not be overlooked.

The effect of biventricular pacing on long term outcomes including mortality is unknown. Hence, the incremental effectiveness and cost effectiveness of this expensive technology remains uncertain. Furthermore, the addition of defibrillation capability to resynchronisation therapy was not addressed in this study.

Despite these limitations, resynchronisation therapy should be considered for patients who have depressed left ventricular systolic function, NYHA class III or IV congestive heart failure symptoms despite optimal medical treatment, and a QRS duration $\geqslant 130 \mathrm{msec}$. Additional data suggest that patients with left bundle-branch block in fact receive greater benefit than those with right bundle-branch block. If longer term data show benefit and additional study allows more precise selection of those patients who will respond, the MIRACLE trial will probably be viewed as a landmark initial step in the development of an effective new treatment method for a small but important subset of patients with advanced congestive heart failure.

William B Hillegass, MD, MPH Andrew E Epstein, MD University of Alabama at Birmingham, Birmingham, Alabama, USA 